\title{
Bond graph based frequency domain sensitivity analysis of multidisciplinary systems
}

\author{
W Borutzky ${ }^{1 *}$ and $\mathbf{J}$ Granda ${ }^{2}$ \\ ${ }^{1}$ Bonn-Rhein-Sieg University of Applied Sciences, Sankt Augustin, Germany \\ ${ }^{2}$ Department of Mechanical Engineering, California State University, Sacramento, California, USA
}

\begin{abstract}
Multidisciplinary systems are described most suitably by bond graphs. In order to determine unnormalized frequency domain sensitivities in symbolic form, this paper proposes to construct in a systematic manner a bond graph from another bond graph, which is called the associated incremental bond graph in this paper. Contrary to other approaches reported in the literature the variables at the bonds of the incremental bond graph are not sensitivities but variations (incremental changes) in the power variables from their nominal values due to parameter changes. Thus their product is power. For linear elements their corresponding model in the incremental bond graph also has a linear characteristic. By deriving the system equations in symbolic state space form from the incremental bond graph in the same way as they are derived from the initial bond graph, the sensitivity matrix of the system can be set up in symbolic form. Its entries are transfer functions depending on the nominal parameter values and on the nominal states and the inputs of the original model. The sensitivities can be determined automatically by the bond graph preprocessor CAMP-G and the widely used program MATLAB together with the Symbolic Toolbox for symbolic mathematical calculation. No particular program is needed for the approach proposed. The initial bond graph model may be non-linear and may contain controlled sources and multiport elements. In that case the sensitivity model is linear time variant and must be solved in the time domain. The rationale and the generality of the proposed approach are presented. For illustration purposes a mechatronic example system, a load positioned by a constant-excitation d.c. motor, is presented and sensitivities are determined in symbolic form by means of CAMP-G/MATLAB.
\end{abstract}

Keywords: first-order frequency domain sensitivities, incremental bond graph, sensitivity matrix in symbolic form

\section{NOTATION}

$\mathbf{A}, \mathbf{B}, \mathbf{C}, \mathbf{D}$
$c$
$E(t)$
$f$
$\mathbf{f}, \mathbf{g}$
$F(s)$
$i$
$I$

$\mathbf{A}, \mathbf{B}, \mathbf{C}, \mathbf{D}$

matrices of a linear time invariant multi-inputs, multi-outputs system flow variable at a port of an element or component voltage of an independent voltage source effort variable at a port of an element or component functions $\mathbf{f}, \mathbf{g}: \mathbf{R}^{\operatorname{dim}(x)+\operatorname{dim}(u)+\operatorname{dim}(p)} \rightarrow \mathrm{R}$ transfer function current inertia parameter

The MS was received on 24 August 2001 and was accepted after revision for publication on 25 September 2001.

* Corresponding author: Bonn-Rhein-Sieg University of Applied Sciences, D-53754 Sankt Augustin, Germany.

\begin{tabular}{|c|c|}
\hline I & $\begin{array}{l}\text { identity matrix of appropriate } \\
\text { dimensions }\end{array}$ \\
\hline$J$ & moment of inertia \\
\hline$k$ & $\begin{array}{l}\text { constant of an effort modulated effort } \\
\text { source }\end{array}$ \\
\hline$k_{\mathrm{T}}$ & $\begin{array}{l}\text { torque constant of a constantly excited } \\
\text { d.c. motor }\end{array}$ \\
\hline$L$ & self-inductance \\
\hline $\mathscr{L} f$ & Laplace transform of a function $f(t)$ \\
\hline$M_{i j}$ & $\begin{array}{l}\text { mutual inductance coefficients of } \\
\text { mutually interacting coils }\end{array}$ \\
\hline$M_{\text {load }}$ & load moment \\
\hline$p$ & $\begin{array}{l}\text { system parameter, generalized } \\
\text { momentum, hydraulic pressure }\end{array}$ \\
\hline$p$ & parameter vector \\
\hline$q$ & vector of generalized displacements \\
\hline$Q$ & hydraulic volume flowrate \\
\hline$r$ & $\begin{array}{l}\text { ratio of a gyrator, number of } \\
\text { parameters }\end{array}$ \\
\hline
\end{tabular}

Proc Instn Mech Engrs Vol 216 Part I: J Systems and Control Engineering 


\begin{tabular}{|c|c|}
\hline$R$ & resistance \\
\hline$s$ & complex variable: $s \in \mathrm{C}$ \\
\hline $\mathbf{S}$ & sensitivity matrix \\
\hline$t$ & time \\
\hline$u$ & $\begin{array}{l}\text { input variable of a single-input, single- } \\
\text { output system }\end{array}$ \\
\hline $\boldsymbol{u}(t)$ & vector of system input variables \\
\hline$x(t)$ & state vector \\
\hline$y$ & system output variable \\
\hline$y(t)$ & vector of system output variables \\
\hline$\Delta$ & determinant of a matrix \\
\hline$\Delta p$ & $\begin{array}{l}\text { deviation of a parameter } p \text { from its } \\
\text { nominal value }\end{array}$ \\
\hline$\Delta P$ & $\begin{array}{l}\text { change in power due to a parameter } \\
\text { change }\end{array}$ \\
\hline$\Delta v$ & $\begin{array}{l}\text { deviation of a variable } v \text { from its } \\
\text { nominal value }\end{array}$ \\
\hline$\lambda$ & flux linkage \\
\hline $\boldsymbol{\Phi}_{C}^{-1}$ & $\begin{array}{l}\text { function } \Phi_{C}^{-1}: \mathrm{R}^{n^{+} r} \rightarrow \mathrm{R} \text { defining the } \\
\text { constitutive relation of an } n \text {-port } \mathrm{C} \text { field } \\
\text { with } r \text { parameters }\end{array}$ \\
\hline$\Phi_{R}$ & $\begin{array}{l}\text { function } \Phi_{R}: \mathrm{R}^{1+r} \rightarrow \mathrm{R} \text { defining the } \\
\text { constitutive relation of a one-port } \\
\text { resistor with } r \text { parameters }\end{array}$ \\
\hline$\omega$ & angular velocity \\
\hline
\end{tabular}

\section{INTRODUCTION}

For the design of reliable systems it is important to know whether a system will still operate as intended when some system parameters vary slightly due to tolerances in the manufacturing process or due to changes in the ambient, e.g. temperature or pressure. This need is obvious, e.g. for integrated circuits. For instance, heat production needs to be taken into account because it affects the operating point of integrated electronic devices since junction diodes depend on the junction temperature.

The sensitivity of the behaviour in the time domain or in the frequency domain with regard to parameter changes is expressed by partial derivatives. If $y$ denotes an output of a lumped parameter system model and $p$ a parameter, e.g. a resistance $R$, then

$$
\frac{\partial y}{\partial p} \frac{p}{y}
$$

is called the relative or normalized sensitivity of $y$ with respect to the parameter $p$. The partial derivative $\partial y / \partial p$ is called the unnormalized sensitivity. The objective in this paper is the determination of unnormalized sensitivities. In the following the adjective unnormalized is omitted. Sensitivities can be calculated in the time domain as well as in the frequency domain. In the second case they are of complex value and depend on the fre- quency $\omega$ in general. In this paper the presentation focuses on frequency domain sensitivities.

A brute force method to determine a sensitivity is to replace the differentials by differences. Performing numerical differentiation, however, has the drawback of poor accuracy because the difference of the computer representations of two nearly equal numbers results in a loss of information. Moreover, it is costly in regard to computation time because at each frequency and for each parameter a simulation run must be performed. To avoid numerical differentiation an approach common in network analysis is to construct a so-called adjoint network $\hat{N}$ from the initial network $N$ and to exploit Tellegen's theorem for an efficient calculation of sensitivities [1]. In the area of bondgraph-based physical system modelling it has been proposed to derive from the bond graph of a system a pseudo bond graph for the sensitivities in which the variables at a bond are sensitivities rather than power variables $[2,3]$.

Instead of representing dependences between sensitivities in a pseudo bond graph, the approach presented in this paper starts from the observation that parameter changes cause perturbed power variables $e+\Delta e$ and $f+\Delta f$ at the ports of an element [4]. Both flow and effort at a port are affected by a parameter variation due to the interaction with the rest of the system. In principle, a parameter change affects all variables in the system. [The variables $e(t)$ and $f(t)$ denote nominal values while $(\Delta e)(t)$ and $(\Delta f)(t)$ denote deviations from nominal values at time $t$.] This paper proposes to derive a true bond graph $G$ for the incremental power variables $\Delta e$ and $\Delta f$ from the initial bond graph $G$. The derived bond graph $\tilde{G}$ is called the associated incremental bond graph. Unnormalized sensitivities can be determined in symbolic form by deriving the system equations from the incremental bond graph. This approach is in accordance with the incremental network approach in network theory. The advantage over an approach that corresponds to the adjoint network approach is that the incremental bond graph provides a better insight into the effect of parameter changes because they are visualized in the incremental bond graph by sources.

The paper is organized in the following manner. In the next section, first-order submodels to be used in the incremental bond graph are developed for linear bond graph elements. An incremental bond graph is constructed by simply replacing the elements by their corresponding submodels. Once the incremental bond graph is available, system equations for the incremental power variables can be derived and by that way unnormalized sensitivities. In Section 3 the case of linear time-invariant systems is considered and illustrated by an example in Section 4 . The presentation continues by extending the approach to bond graphs with non-linear multiport 
elements (Section 5). Finally, the results are summarized and some conclusions are drawn.

\section{INCREMENTAL MODELS CORRESPONDING TO THE BOND GRAPH ELEMENTS}

In order to keep the presentation easy to survey, in this section, sources, stores and resistors are assumed to be one-port elements with a linear characteristic, while transformers and gyrators are assumed to have two ports. In the following $t \in \mathrm{R}, t>0$, has the meaning of time. Power variables $e(t)$ and $f(t)$, as well as the parameter in the linear constitutive relation between the power variables of a port, denote nominal values. Deviations from the nominal values of power variables are denoted by $(\Delta e)(t)$ and $(\Delta f)(t)$. They may have any value in $\mathrm{R}$ and are not limited to positive values. In order to avoid an overloading of variable names by indices, an index $n$ meaning nominal is dropped.

Consider a linear resistor with the resistance $R$ :

$$
e_{R}(t)-R f_{R}(t)=0
$$

The basic idea is that a variation $\Delta R$ in the resistance causes perturbed power variables $\left(e_{R}+\Delta e_{R}\right)(t)$, and $\left(f_{R}+\Delta_{R}\right)(t)$ at the power port:

$$
\left(e_{R}+\Delta e_{R}\right)(t)-(R+\Delta R)\left(f_{R}+\Delta f_{R}\right)(t)=0
$$

Substituting equation (1) into equation (2) and neglecting the higher-order term $\Delta R \Delta f_{R}$ a linear relation between the incremental power variables $\Delta e_{R}$ and $\Delta f_{R}$ is obtained:

$$
\begin{aligned}
\left(\Delta e_{R}\right)(t) & =R\left(\Delta f_{R}\right)(t)+f_{R}(t) \Delta R \\
& =R\left(\Delta f_{R}\right)(t)+\frac{\partial e_{R}}{\partial R}(t) \Delta R
\end{aligned}
$$

Equation (3) may be interpreted as a resistor with power port variables $\Delta e$ and $\Delta f$ and a modulated effort sink attached to a 1-junction (Fig. 1). The sink represents the effect of the parameter change $\Delta R$. It is modulated by the nominal power variable $\left(\partial e_{R} / \partial R\right)(t)=f_{R}(t)$ in the initial bond graph. As can be seen from Fig. 1 the incremental model corresponding to a resistor differs

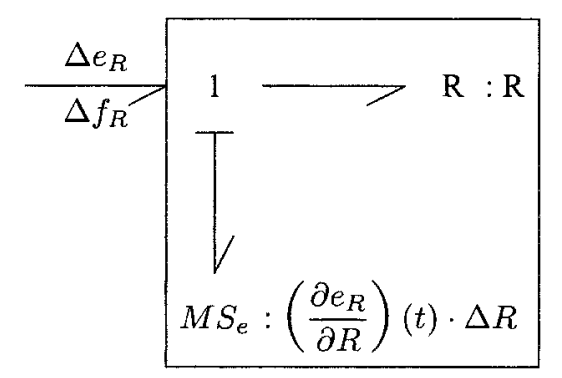

Fig. 1 Submodel in the incremental bond graph corresponding to a resistor from the $\mathrm{R}$ element simply by an additional sink which does not affect causalities in the initial bond graph.

Note that the product $\Delta e_{R} \Delta f_{R}$ is only a fraction of the power change $\Delta P$ due to the parameter change $\Delta R$

$$
\begin{aligned}
\Delta P & =\left(e_{R}+\Delta e_{R}\right)\left(f_{R}+\Delta f_{R}\right)-e_{R} f_{R} \\
& =\Delta e_{R} \Delta f_{R}+\text { further terms }
\end{aligned}
$$

For both types of one-port stores a similar result is obtained. Consider, for instance, an inertia. A change $\Delta I$ in the inertia parameter results in perturbed power variables at its ports and consequently in a perturbed generalized momentum $p+\Delta p$ :

$$
\left(f_{I}+\Delta f_{I}\right)(t)=\frac{1}{I+\Delta I}(p+\Delta p)(t)
$$

Again, neglecting higher-order terms $\Delta I \Delta f_{I}$ a sum of incremental flows is obtained

$$
\begin{aligned}
\left(\Delta f_{I}\right)(t) & =\frac{(\Delta p)(t)}{I}-\frac{\Delta I}{I} f_{I}(t) \\
& =\frac{(\Delta p)(t)}{I}+\left(\frac{\partial f_{I}}{\partial I}\right)(t) \Delta I
\end{aligned}
$$

that may be displayed by a bond graph as depicted in Fig. 2. As for the incremental model of an $\mathrm{R}$ element, the additional sink in the incremental model of a store modulated by the sensitivity of the flow $f_{I}$ with respect to the store parameter $\left(\partial f_{I} / \partial I\right)(t)=\left(f_{I} / I\right)(t)$ does not affect causalities. However, contrary to a store in the initial bond graph its corresponding submodel in the incremental bond graph is not energy conservative due to the sink which reflects the change in the store parameter. In the following the program CAMP-G/MATLAB is used. For convenience the sink in Fig. 2 is split into an independent sink that represents the change in the inertia parameter $\Delta I$ and a modulated transformer (Fig. 3).

As for a resistor and a store, an incremental bond graph may be developed for a two-port transformer and a two-port gyrator in the same way. The result for a gyrator is depicted in Fig. 4. Because of the two sinks the incremental model corresponding to a gyrator is not power conservative. Since the constitutive relations for 1 - and 0 -junctions are linear, they hold also for the

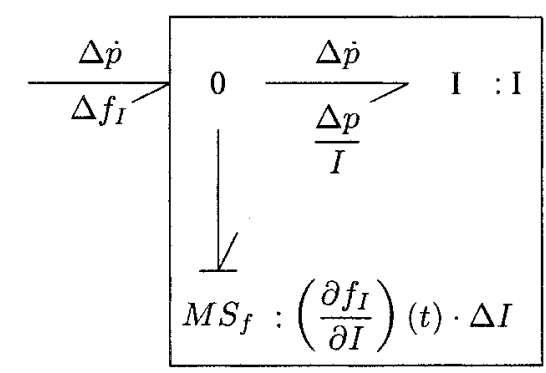

Fig. 2 Submodel in the incremental bond graph corresponding to an inertia 


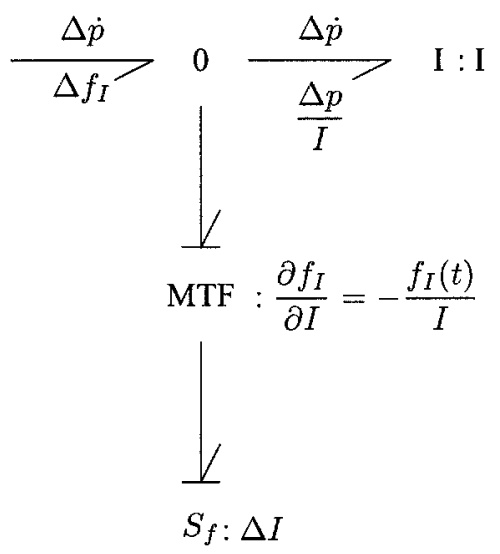

Fig. 3 Incremental bond graph of an inertia

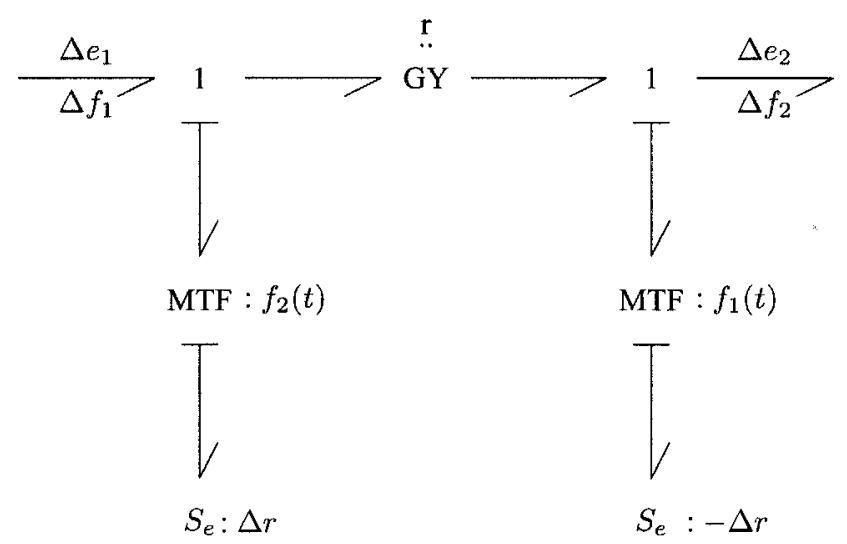

Fig. 4 Incremental bond graph of a gyrator

incremental power variables; i.e. the incremental bond graph which corresponds to a 0 -junction again is a 0 -junction.

Finally, for example, an effort-modulated effort source is to be replaced by an incremental bond graph shown in Fig. 5. The incremental model that corresponds to an independent source is a source of the same type with vanishing output. Having determined for each basic bond graph element a model that relates the incremental power variables, it is easy to construct the associated incremental bond graph from a given bond graph. The elements are replaced by their corresponding incremental bond graphs. 0 - and 1 -junctions remain the same for the incremental power variables. The incremental models of

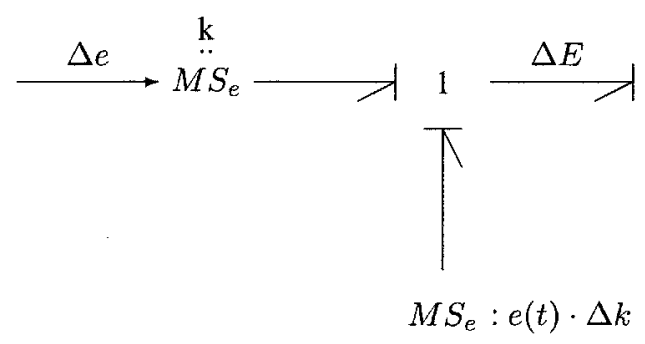

Fig. 5 Incremental bond graph of an effort-modulated effort source the other bond graph elements differ from the original element only by an additional sink. Hence, the structure of the associated incremental bond graph differs from that of the initial bond graph only by the additional sinks which express explicitly the effect of parameter changes. Since the independent sources transform into sources of the same type with vanishing output, the resulting associated incremental bond graph retains the structure of the original bond graph but with different sources at different locations. If all elements in an initial bond graph are replaced by their corresponding incremental model, the resulting incremental system bond graph accounts for all parameter changes. Owing to the linearity of the junction structure the result of changes in several parameters is the superposition of all effects due to a change in a single parameter. Consequently, if the effect of a change in only one parameter is of interest, it is sufficient to replace only the affected element by its incremental model. In that way, computation time may be saved in the determination of the required sensitivity from the incremental system bond graph.

\section{DERIVING FREQUENCY DOMAIN SENSITIVITIES FROM THE INCREMENTAL BOND GRAPH}

A linear time-invariant system is described by the two vector equations with the state vector $\boldsymbol{x}(t)$, the vector $\boldsymbol{u}(t)$ of system inputs, the vector $\boldsymbol{y}(t)$ of outputs and matrices $\mathbf{A}, \mathbf{B}, \mathbf{C}$ and $\mathbf{D}$ of appropriate dimensions. These matrices depend on the time invariant parameter vector $\boldsymbol{p}$ :

$$
\begin{aligned}
& \dot{\boldsymbol{x}}(t)=\mathbf{A}(\boldsymbol{p}) \boldsymbol{x}(t)+\mathbf{B}(\boldsymbol{p}) \boldsymbol{u}(t) \\
& \boldsymbol{y}(t)=\mathbf{C}(\boldsymbol{p}) \boldsymbol{x}(t)+\mathbf{D}(\boldsymbol{p}) \boldsymbol{u}(t)
\end{aligned}
$$

As is known, the matrices $\mathbf{A}, \mathbf{B}, \mathbf{C}$ and $\mathbf{D}$ can be built by means of submatrices of the junction structure matrix and the constitutive equations of the elements [5]. Another method proposed in reference [6] is to use a computer-generated approach directly from a graphical input of the input of the bond graph. Since the incremental bond graph differs from the initial bond graph only by the sources that express parameter changes, the equations of the incremental bond graph are

$$
\begin{aligned}
\Delta \dot{\boldsymbol{x}}(t) & =\mathbf{A}(\boldsymbol{p}) \Delta \boldsymbol{x}(t)+\tilde{\mathbf{B}}(\boldsymbol{x}(t), \boldsymbol{u}(t), \boldsymbol{p}) \Delta \boldsymbol{p} \\
\Delta \boldsymbol{y}(t) & =\mathbf{C}(\boldsymbol{p}) \Delta \boldsymbol{x}(t)+\tilde{\mathbf{D}}(\boldsymbol{x}(t), \boldsymbol{u}(t), \boldsymbol{p}) \Delta \boldsymbol{p}
\end{aligned}
$$

In these equations, $\Delta_{\boldsymbol{p}}$ denotes the parameter changes. The matrices $\tilde{\mathbf{B}}$ and $\tilde{\mathbf{D}}$ depend on the nominal vectors $\boldsymbol{x}(t)$ and $\boldsymbol{u}(t)$ satisfying equations (7a) and (7b) and the parameter vector $\boldsymbol{p}$. Consequently, they depend on time. If $s \in \mathrm{C}$, then the Laplace transform yields

$$
(s \mathbf{I}-\mathbf{A}) \mathscr{L} \boldsymbol{x}=\mathbf{B} \mathscr{L} \boldsymbol{u}
$$


and

$$
(s \mathbf{I}-\mathbf{A})(\mathscr{L} \Delta \boldsymbol{x})(s)=(\mathscr{L} \tilde{\mathbf{B}})(s) \Delta \boldsymbol{p}
$$

Once the LU factors of the matrix $(s \mathbf{I}-\mathbf{A})$ are known, they can be used to solve for $\mathscr{L} \boldsymbol{x}$ and again to solve for $\mathscr{L} \Delta \boldsymbol{x}$. By using the LU factors twice the computational effort is greatly reduced. If $\mathscr{L} \Delta \boldsymbol{x}$ is substituted into the Laplace transform of equation (8b), the following equation is obtained:

$$
(\mathscr{L} \Delta \boldsymbol{y})(s)=\left[\mathbf{C}(s \mathbf{I}-\mathbf{A})^{-1} \mathscr{L} \tilde{\mathbf{B}}+\mathscr{L} \tilde{\mathbf{D}}\right](s) \Delta \boldsymbol{p}
$$

Assuming infinitesimal small parameter changes, the matrix in equation (11) is just the sensitivity matrix

$$
\mathbf{S}(s):=\frac{\partial \mathscr{L} \boldsymbol{y}}{\partial \boldsymbol{p}}=\left[\mathbf{C}(s \mathbf{I}-\mathbf{A})^{-1} \mathscr{\mathscr { B }} \tilde{\mathbf{B}}+\mathscr{L} \tilde{\mathbf{D}}\right](s)
$$

to be determined.

Note that in equations (7a) and (7b) the entries in the matrices depend on the components of the parameter vector $\boldsymbol{p}$. In general these dependences are non-linear. By building the total differentials $\mathrm{d} \dot{\boldsymbol{x}}$ and $\mathrm{d} \boldsymbol{y}$ from equations (7a) and (7b) and taking into account that $\mathrm{d} \boldsymbol{u}$ vanishes, the matrices $\tilde{\mathbf{B}}$ and $\tilde{\mathbf{D}}$ are obtained:

$$
\begin{aligned}
\tilde{\mathbf{B}}(t) & =\frac{\partial}{\partial \boldsymbol{p}}(\mathbf{A} \boldsymbol{x}+\mathbf{B} \boldsymbol{u})(t) \\
\tilde{\mathbf{D}}(t) & =\frac{\partial}{\partial \boldsymbol{p}}(\mathbf{C} \boldsymbol{x}+\mathbf{D} \boldsymbol{u})(t)
\end{aligned}
$$

Thus, once the equations (7a) and (7b) have been deduced from the initial bond graph, the matrices $\tilde{\mathbf{B}}$ and $\tilde{\mathbf{D}}$ can be computed by symbolic differentiation with respect to the parameters according to equations (13a) and (13b). After Laplace transform of equation (7a), $\mathscr{L} \boldsymbol{x}$ can be expressed by $\mathscr{L} \boldsymbol{u}$; i.e. the matrices $\mathscr{L} \tilde{\mathbf{B}}$ and $\mathscr{L} \tilde{\mathbf{C}}$ depend on $\mathscr{L} \boldsymbol{u}$ and the constant parameter vector $\boldsymbol{p}$. Finally, the sensitivity matrix is computed according to equation (12) as a function of $\boldsymbol{p}$ and $\mathscr{L} \boldsymbol{u}$.

Alternatively, the equations for the incremental power variables may be derived from the associated incremental bond graph. Assuming infinitesimal small increments the coefficients in the equations are the sensitivities to be determined.

Note that the ratio of an incremental variable $\mathscr{L} \Delta y$ to a parameter change $\Delta p$ is a transfer function between the output variable $\mathscr{L} \Delta y$ and the input variable $\Delta p$, which essentially is the output of a modulated source in the incremental bond graph. As Brown [7] has shown, transfer functions can be determined by applying Mason's rule directly to the bond graph. Alternatively, programs, e.g. the bond graph preprocessor CAMP-G and the widely used general-purpose program MATLAB for numerical and symbolic calculations, can derive transfer functions in symbolic form automatically from a bond graph by setting up the state space model and transforming it into a matrix of transfer functions $[8,9]$. The essential point is that these transfer functions are the unnormalized sensitivities to be determined if incremental changes are assumed to be small.

In the following section the entire process is illustrated. First CAMP-G is used to enter the incremental bond graph of an electrical motor and to generate a MATLAB procedure. In a second step, MATLAB is used to produce the sensitivity matrix $\mathbf{S}$.

\section{AN EXAMPLE}

For an illustration of the approach described so far consider the simple example of a d.c. motor with constant excitation. The schematic diagram of the positioning system is shown in Fig. 6. The corresponding bond graph is depicted in Fig. 7. In that bond graph, $M_{\text {load }}$ denotes an external disturbing load moment. From the bond graph the following state equations can be derived:

$$
\frac{\mathrm{d}}{\mathrm{d} t}\left[\begin{array}{c}
p_{\mathrm{m}} \\
p_{\mathrm{e}}
\end{array}\right]=\underbrace{\left[\begin{array}{cc}
-\frac{R_{\mathrm{m}}}{J_{\mathrm{m}}} & \frac{k_{\mathrm{T}}}{L_{\mathrm{a}}} \\
-\frac{k_{\mathrm{T}}}{J_{\mathrm{m}}} & -\frac{R_{\mathrm{a}}}{L_{\mathrm{a}}}
\end{array}\right]}_{\mathbf{A}} \underbrace{\left[\begin{array}{c}
p_{\mathrm{m}} \\
p_{\mathrm{e}}
\end{array}\right]}_{\boldsymbol{x}}+\underbrace{\left(\begin{array}{cc}
0 & 1 \\
1 & 0
\end{array}\right)}_{\mathbf{B}} \underbrace{\left[\begin{array}{c}
E \\
M_{\text {load }}
\end{array}\right]}_{\boldsymbol{u}}
$$

If the current through the armature, $i_{\mathrm{a}}$, is chosen as an output variable $y$, the matrices $\mathbf{C}$ and $\mathbf{D}$ become

$$
\underbrace{\left[i_{\mathrm{a}}\right]}_{\mathbf{y}}=\underbrace{\left(\begin{array}{ll}
0 & \frac{1}{L_{\mathrm{a}}}
\end{array}\right)}_{\mathbf{C}}\left[\begin{array}{c}
p_{\mathrm{m}} \\
p_{\mathrm{e}}
\end{array}\right]+\underbrace{\left(\begin{array}{ll}
0 & 0
\end{array}\right)}_{\mathbf{D}}\left[\begin{array}{c}
E \\
M_{\mathrm{load}}
\end{array}\right]
$$

According to equations (13a) the matrix $\tilde{\mathbf{B}}$ is

$$
\begin{aligned}
& \tilde{\mathbf{B}}=\frac{\partial}{\partial \boldsymbol{p}}(\mathbf{A} \boldsymbol{x}+\mathbf{B} \boldsymbol{u}) \\
& =\frac{\partial}{\partial \boldsymbol{p}}(\mathbf{A} \boldsymbol{x}) \\
& =\frac{\partial}{\partial\left(R_{\mathrm{a}}, L_{\mathrm{a}}, k_{\mathrm{T}}, R_{\mathrm{m}}, J_{\mathrm{m}}\right)}\left[\begin{array}{c}
-\frac{R_{\mathrm{m}}}{J_{\mathrm{m}}} p_{\mathrm{m}}+\frac{k_{\mathrm{T}}}{L_{\mathrm{a}}} p_{\mathrm{e}} \\
-\frac{k_{\mathrm{T}}}{J_{\mathrm{m}}} p_{\mathrm{m}}-\frac{R_{\mathrm{a}}}{L_{\mathrm{a}}} p_{\mathrm{e}}
\end{array}\right] \\
& =\left(\begin{array}{ccccc}
0 & -\frac{k_{\mathrm{T}}}{L_{\mathrm{a}}^{2}} p_{\mathrm{e}} & \frac{1}{L_{\mathrm{a}}} p_{\mathrm{e}} & -\frac{1}{J_{\mathrm{m}}} p_{\mathrm{m}} & \frac{R_{\mathrm{m}}}{J_{\mathrm{m}}^{2}} p_{\mathrm{m}} \\
-\frac{1}{L_{\mathrm{a}}} p_{\mathrm{e}} & \frac{R_{\mathrm{a}}}{L_{\mathrm{a}}^{2}} p_{\mathrm{e}} & -\frac{1}{J_{\mathrm{m}}} p_{\mathrm{m}} & 0 & \frac{k_{\mathrm{T}}}{J_{\mathrm{m}}^{2}} p_{\mathrm{m}}
\end{array}\right) \\
& =\frac{\partial \mathbf{A}}{\partial \boldsymbol{p}} \boldsymbol{x}
\end{aligned}
$$




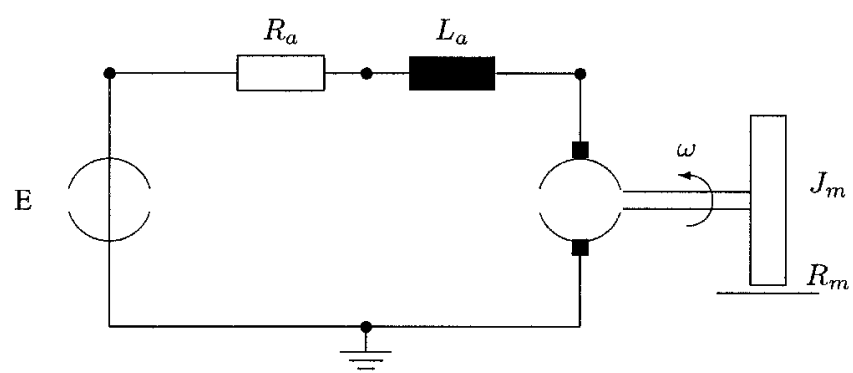

Fig. 6 Positioning system with constantly excited d.c. motor

According to equation (13b) the matrix $\tilde{\mathbf{D}}$ becomes

$$
\begin{aligned}
& \tilde{\mathbf{D}}=\frac{\partial}{\partial \boldsymbol{p}}(\mathbf{C} \boldsymbol{x}+\mathbf{D} \boldsymbol{u}) \\
& =\frac{\partial}{\partial \boldsymbol{p}}(\mathbf{C} \boldsymbol{x}) \\
& =\frac{\partial}{\partial\left(R_{\mathrm{a}}, L_{\mathrm{a}}, k_{\mathrm{T}}, R_{\mathrm{m}}, J_{\mathrm{m}}\right)}\left(\frac{1}{L_{\mathrm{a}}} p_{\mathrm{e}}\right) \\
& =\left(\begin{array}{lllll}
0 & -\frac{1}{L_{\mathrm{a}}^{2}} p_{\mathrm{e}} & 0 & 0 & 0
\end{array}\right) \\
& =\frac{\partial \mathbf{C}}{\partial \boldsymbol{p}} \boldsymbol{x}
\end{aligned}
$$

The matrix $(s \mathbf{I}-\mathbf{A})^{-1}$ becomes

$$
(s \mathbf{I}-\mathbf{A})^{-1}=\frac{1}{\Delta}\left(\begin{array}{cc}
s+\frac{R_{\mathrm{a}}}{L_{\mathrm{a}}} & \frac{k_{\mathrm{T}}}{L_{\mathrm{a}}} \\
-\frac{k_{\mathrm{T}}}{J_{\mathrm{m}}} & s+\frac{R_{\mathrm{m}}}{J_{\mathrm{m}}}
\end{array}\right)
$$

with

$$
\Delta:=s^{2}+\left(\frac{R_{\mathrm{a}}}{L_{\mathrm{a}}}+\frac{R_{\mathrm{m}}}{J_{\mathrm{m}}}\right) s+\frac{R_{\mathrm{m}} R_{\mathrm{a}}}{J_{\mathrm{m}} L_{\mathrm{a}}}+\frac{k_{\mathrm{T}}^{2}}{J_{\mathrm{m}} L_{\mathrm{a}}}
$$

From the above matrices a lengthy expression, not given here, is obtained for the sensitivity matrix

$$
\mathbf{S}=\mathbf{C}(s \mathbf{I}-\mathbf{A})^{-1} \mathscr{L} \tilde{\mathbf{B}}+\mathscr{L} \tilde{\mathbf{D}}
$$

Alternatively, the associated incremental bond graph may be entered into the program CAMP-G (Fig. 8). CAMP-G can generate a file campgsym which directs
MATLAB to derive the matrices $\mathbf{A}, \mathbf{C}, \tilde{\mathbf{B}}$ and $\tilde{\mathbf{D}}$, and any sensitivities that are required. Note that in Fig. 8 the two sinks in the incremental model of the gyrator have been combined into one. The file campgsym must be edited in appropriate places according to the choice of the output variable $\boldsymbol{y}$. If the file is invoked at the MATLAB prompt, the program produces the same matrices as above and the sensitivity matrix given in Fig. 9. For CAMP-G/MATLAB the incremental bond graph entered is just an ordinary bond graph for which MATLAB generates the transfer matrix $\mathbf{H}$. However, since the sinks in the incremental bond graph reflect the parameter changes, the resulting transfer matrix is the sensitivity matrix to be set up. Note that bonds are labelled by numbers; variable names and parameter names are not used as annotations by CAMP-G. For instance, the parameter of the gyrator is not denoted by the torque constant $k_{\mathrm{T}}$, but by means of the numbers of the incident bonds. In CAMP-G the gyrator ratio is $1 / G 8 x 9$. Taking into account the notation used in CAMP-G it can be shown that for instance the characteristic polynomial in Fig. 9 is indeed the determinant $\Delta$ of $(s \mathbf{I}-\mathbf{A})^{-1}$ given by equation (19).

As has been mentioned by the end of Section 2, computation simplifies if the effect of a change in only one parameter is of interest. Then only one element must be replaced by its associated incremental submodel. Suppose that there is a change in mechanical friction. Its effect on the current into the voltage source will be studied. The incremental bond graph for that case is given in Fig. 10. From the incremental bond graph in Fig. 10 the following two equations can be immediately derived.

$$
s \mathscr{L} \Delta i=\frac{1}{L_{\mathrm{a}}}\left(-R_{\mathrm{a}} \mathscr{L} \Delta i-k_{\mathrm{T}} \mathscr{L} \Delta \omega\right)
$$

and

$$
s \mathscr{L} \Delta \omega=\frac{1}{J_{\mathrm{m}}}\left[k_{\mathrm{T}} \mathscr{\mathscr { L }} \Delta i-R_{\mathrm{m}} \mathscr{\mathscr { L }} \Delta \omega-\left(\mathscr{L} f_{R_{\mathrm{m}}}\right) \Delta R_{\mathrm{m}}\right]
$$

From equations (21) and (22) the sensitivity $\partial \mathscr{L} i / \partial R_{\mathrm{m}}$
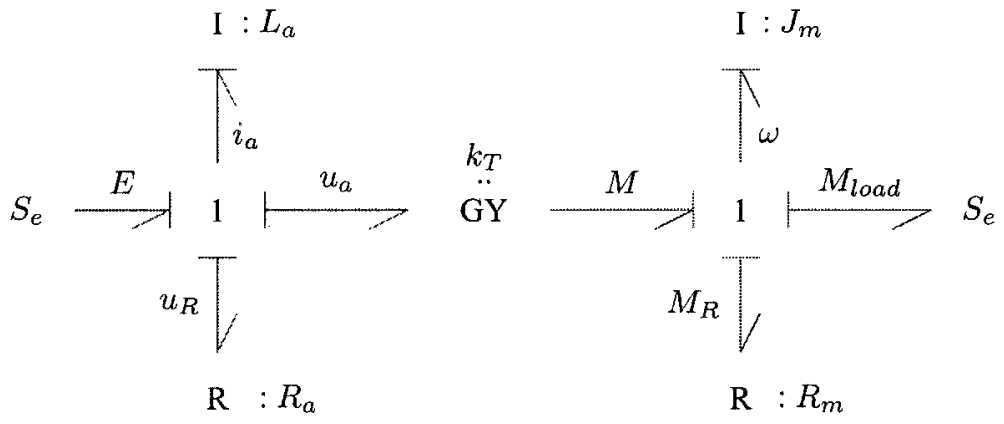

Fig. 7 Bond graph of the positioning system 


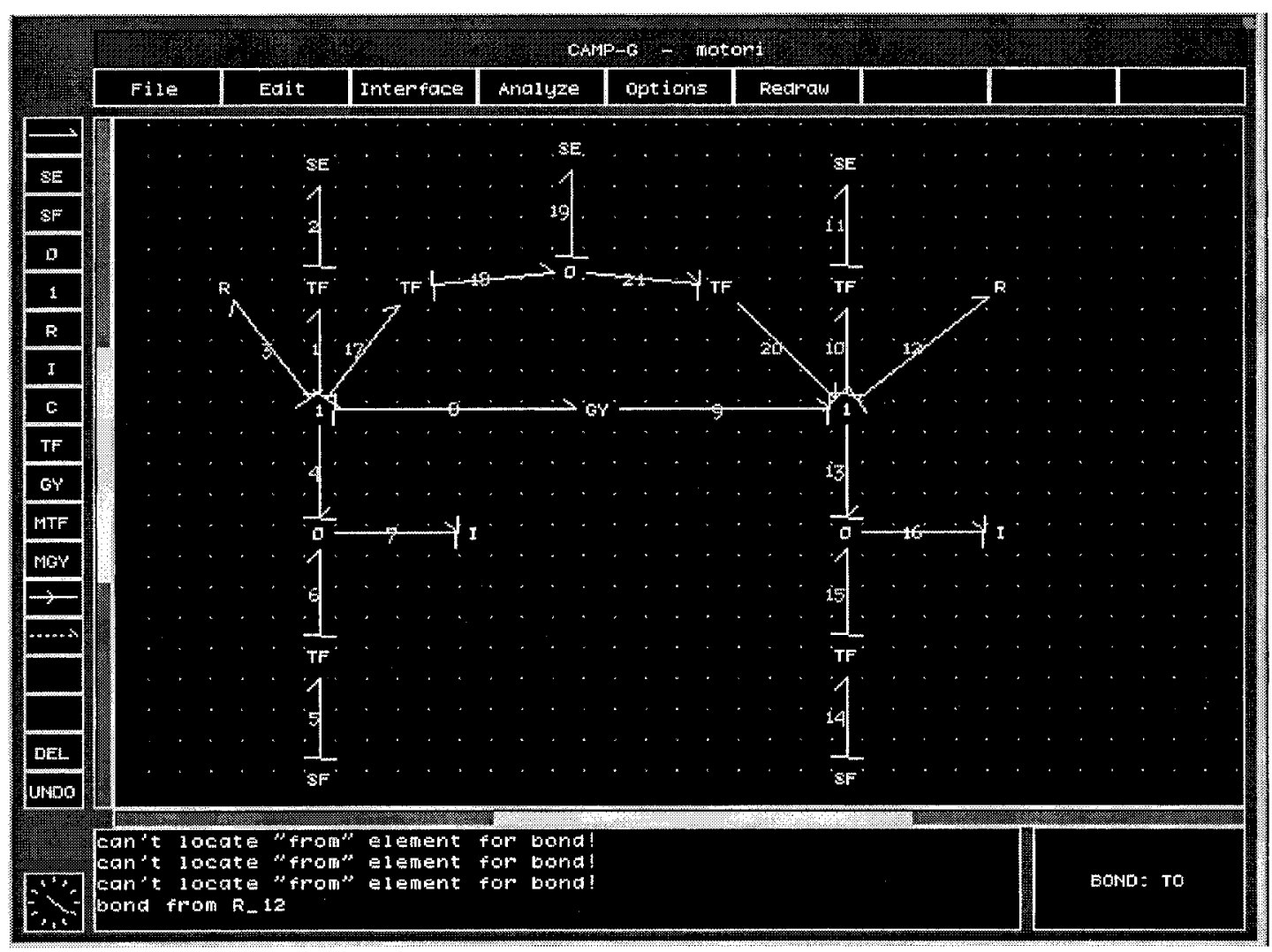

Fig. 8 Associated incremental bond graph for the positioning system

Characteristic Polynomial

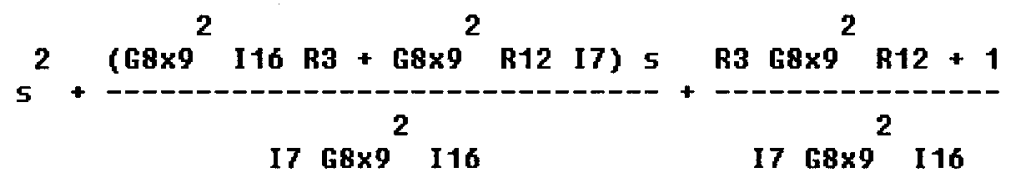

... Transfer Functions Matrix H ...
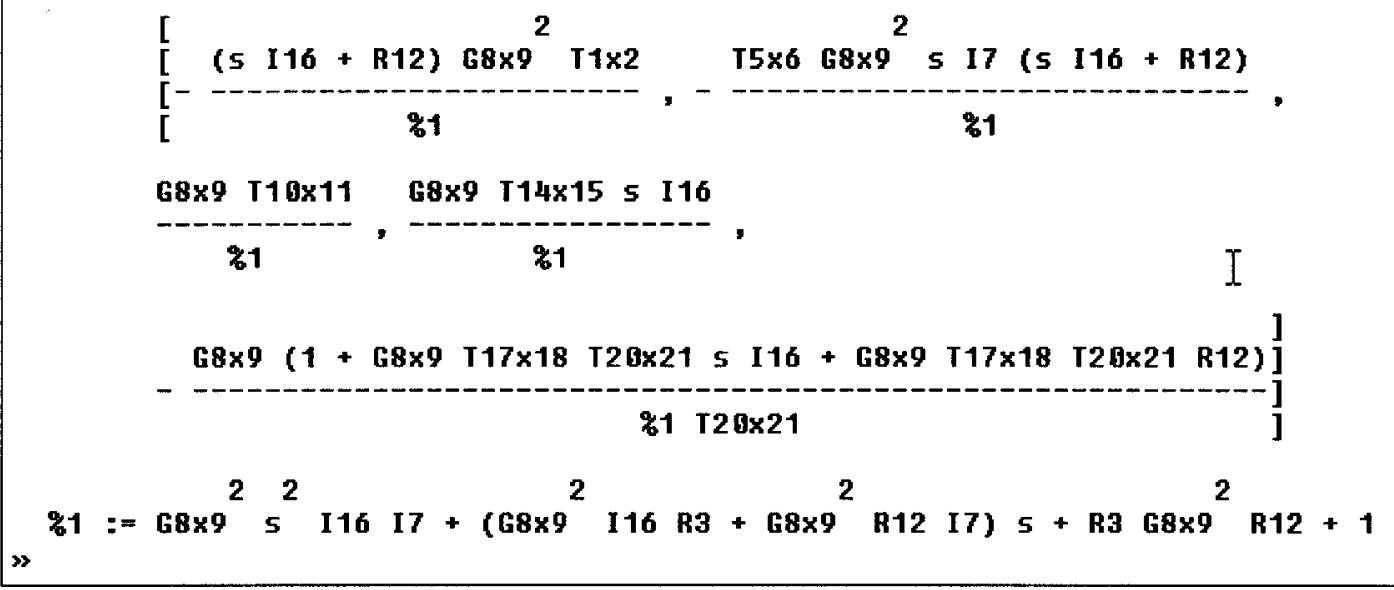

Fig. 9 Sensitivity matrix of the positioning system produced by MATLAB 


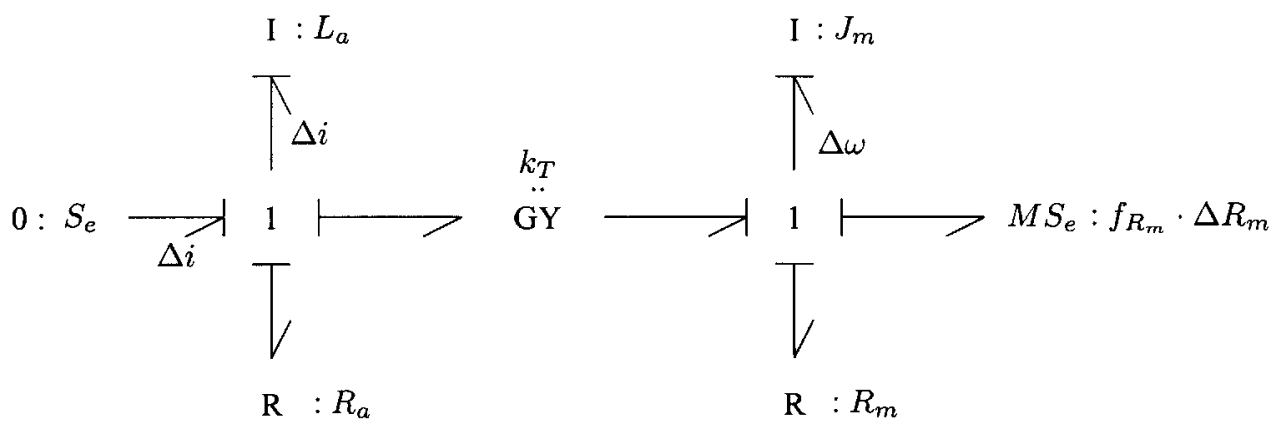

Fig. 10 Sensitivity bond graph for the case of a deviation in mechanical friction

is obtained after some steps:

$$
\frac{\partial \mathscr{L} i}{\partial R_{\mathrm{m}}}=\frac{\left[k_{\mathrm{T}} /\left(L_{\mathrm{a}} J_{\mathrm{m}}\right)\right] \mathscr{L} f_{R_{\mathrm{m}}}}{\left(s+R_{\mathrm{a}} / L_{\mathrm{a}}\right)\left(s+R_{\mathrm{m}} / J_{\mathrm{m}}\right)+k_{\mathrm{T}}^{2} /\left(L_{\mathrm{a}} J_{\mathrm{m}}\right)}
$$

In equation (23) the denominator is the determinant given by equation (19). An equivalent expression to that in equation (23) is obtained if the incremental bond graph of Fig. 10 is entered in CAMP-G and the procedure campgsym generated by CAMP-G is run in MATLAB. Figure 11 shows how bonds are labelled by CAMP-G. The generated sensitivity is shown in Fig. 12 .

The approach presented so far may be applied also to models in which switches are represented by a Booleancontrolled modulated transformer and a resistor accounting for the ON state resistance.

\section{EXTENSION TO NON-LINEAR MULTIPORT ELEMENTS}

\subsection{Non-linear one-port elements}

The approach presented so far is not limited to linear one-port elements, nor elements with one single parameter. As a simple example, consider a hydraulic orifice. Its static behaviour is given by the well-known square

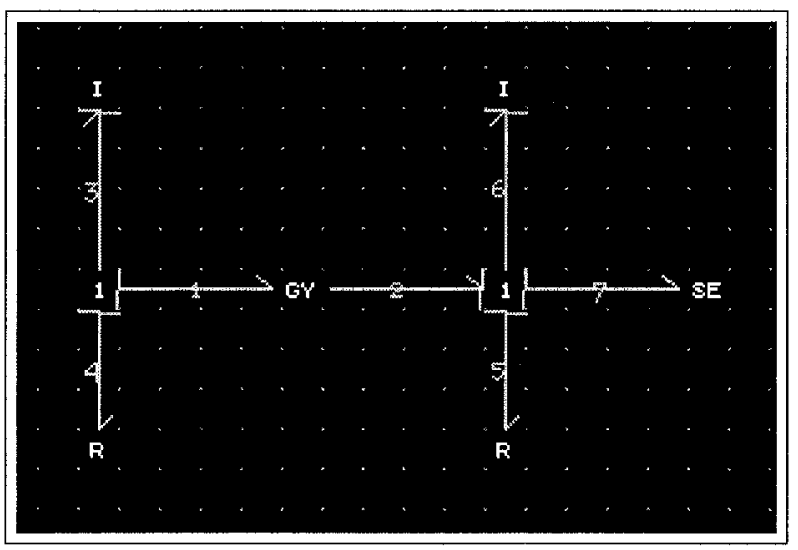

Fig. 11 Sensitivity bond graph entered in CAMP-G root law:

$$
Q=c_{\mathrm{d}} A \sqrt{\frac{2}{\rho}|\Delta p|} \operatorname{sgn}(\Delta p)
$$

In equation (24) the parameter $c_{\mathrm{d}}$ denotes the discharge coefficient and $A$ the cross-sectional area. The equation not only is a non-linear relation between the hydraulic power variables $Q$ and $\Delta p$ but also includes the crosssectional area $A$ which may depend on the position $x$ of the spool in spool valves; i.e. the element may depend not only on parameters that may vary but also on control signals determined by other parts of the model. (Moreover, for small pressure drops the discharge coefficient $c_{\mathrm{d}}$ is not a constant, but a non-linear function of the volume flowrate $Q$ which contains another parameter, namely the oil viscosity.)

By taking a closer look at the formulae of the incremental models derived in Section 2 it can be seen that they may be obtained by building total differentials. Hence, suppose a non-linear one-port resistor is given by the constitutive equation

$$
e_{R}(t)=\Phi_{R}\left(f_{R}(t), \boldsymbol{p}\right)
$$

with a constant parameter vector $\boldsymbol{p}$. In the following all non-linear functions are assumed to be differentiable. Building the total differential of $e_{R}$ immediately yields the relation between the small deviations of the power port variables from nominal values due to changes in the parameters of the resistor:

$$
\Delta e_{R}(t)=\frac{\partial \Phi_{R}}{\partial f_{R}}\left(f_{R}(t), \boldsymbol{p}\right) \Delta f_{R}(t)+\frac{\partial \Phi_{R}}{\partial \boldsymbol{p}}\left(f_{R}(t), \boldsymbol{p}\right) \Delta \boldsymbol{p}
$$

Thus, while the original model may be non-linear, the associated incremental model is linear time variant. As in the case of a linear resistor, equation (26) may be represented by a resistor with a (time-dependent) resistance and a modulated effort sink attached to a 1 -junction. The 1-junction represents the incremental flow $\Delta f_{R}$ (Fig. 13). In practice it may happen that, although an element or a component model has several parameters, the effect of a change in only one parameter 


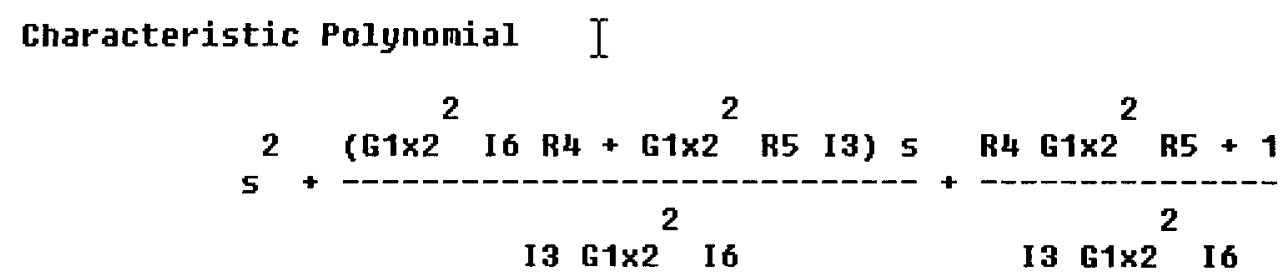

... Transfer Functions Matrix H ...

G1x2

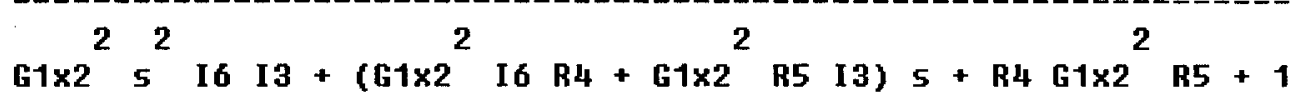

Fig. 12 Sensitivity $\left(1 / \mathscr{L} f_{R_{\mathrm{m}}}\right)\left(\partial \mathscr{L} i / \partial R_{\mathrm{m}}\right)$ generated by MATLAB

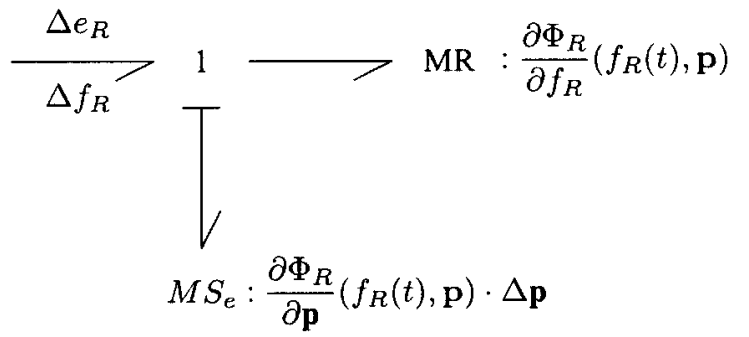

Fig. 13 Incremental model of a non-linear one-port resistor

is of interest. In that case the second term of equation (26) reduces to a product of two scalars.

Although the objective in this article is sensitivities rather than the development of a bond graph for the linearized system, the procedure is similar to that adopted by Karnopp [10]. In fact, the work on sensitivity analysis presented in this article has been inspired by the previous work of Karnopp on the bond graph representation of linearized systems. For instance, in reference [10] the bonds of the bond graph representing the linearized system carry small deviations of the power variables from their steady state values and the product of these deviations is only a part of the power conveyed between two ports in the initial bond graph model.

\subsection{Multiports}

Bond graph elements may depend on more than one parameter. They may also have more than one power variable as input; i.e. they may be multiport elements. An infinitesimal small variation in the output power variables due to small perturbations in the parameters again is obtained through derivation of the total differential. As an example, consider a non-linear multiport $\mathrm{C}$ store, e.g. a movable-plate capacitor, with the constitutive equation

$$
e_{C}(t)=\boldsymbol{\Phi}_{C}^{-1}(\boldsymbol{q}(t), \boldsymbol{p})
$$

I06101 @ IMechE 2002 in which $\boldsymbol{q}(t)$ denotes the generalized displacement and $\boldsymbol{p}$ a constant parameter vector. Infinitesimally small parameter changes result in the linear time-variant

$$
\Delta e_{C}(t)=\frac{\partial \boldsymbol{\Phi}_{C}^{-1}}{\partial \boldsymbol{q}}(\boldsymbol{q}(t), \boldsymbol{p}) \Delta \boldsymbol{q}(t)+\frac{\partial \boldsymbol{\Phi}_{C}^{-1}}{\partial \boldsymbol{p}}(\boldsymbol{q}(t), \boldsymbol{p}) \Delta \boldsymbol{p}
$$

Again, equation (28) may be represented in a multibond graph by a $\mathrm{C}$ store and a sink attached to an array of 1-junctions, or it may be depicted equivalently by means of single bonds, as shown in Fig. 14. A similar result is obtained for multiport I elements.

\subsection{Models containing non-linear multiports}

If the constitutive relations of a multiport are nonlinear, the constitutive relations of the corresponding

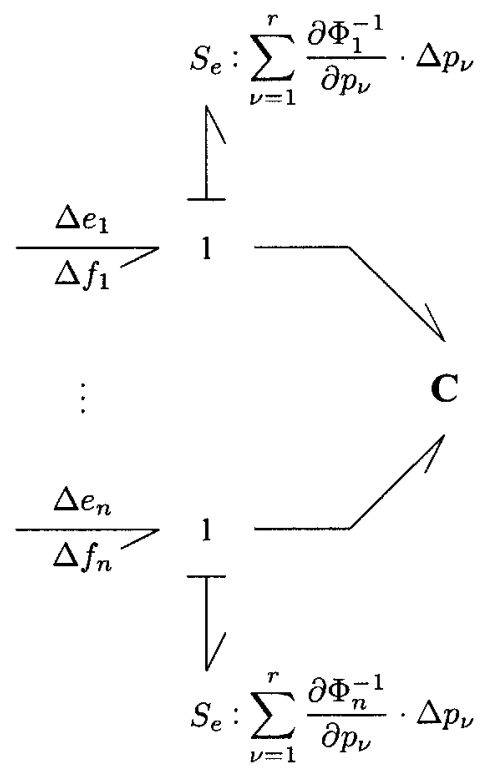

Fig. 14 Incremental model of a non-linear multiport $C$ store Proc Instn Mech Engrs Vol 216 Part I: J Systems and Control Engineering 
incremental model are linear time variant [cf. equations (26) and (28)]. Consequently, in that case, equations deduced from the incremental bond graph cannot be transformed into the frequency domain by means of the Laplace transform. However, equations can be deduced that determine the state sensitivities $\partial \boldsymbol{x} / \partial \boldsymbol{p}$ and the output sensitivities $\partial \boldsymbol{y} / \partial \boldsymbol{p}$ as functions of time provided that $\boldsymbol{x}(t)$ and $\boldsymbol{u}(t)$ of the original model are known; i.e. the equations for $\boldsymbol{x}(t)$ and the sensitivities $\partial \boldsymbol{x} / \partial \boldsymbol{p}$ and $\partial \boldsymbol{y} / \partial \boldsymbol{p}$ must be solved simultaneously.

From the original bond graph equations,

$$
\begin{aligned}
& \dot{\boldsymbol{x}}(t)=\boldsymbol{f}(\boldsymbol{x}(t), \boldsymbol{u}(t), \boldsymbol{p}) \\
& \boldsymbol{y}(t)=\boldsymbol{g}(\boldsymbol{x}(t), \boldsymbol{u}(t), \boldsymbol{p})
\end{aligned}
$$

can be derived. The incremental bond graph model provides the equations

$$
\begin{aligned}
& \Delta \dot{\boldsymbol{x}}(t)=\frac{\partial \boldsymbol{f}}{\partial \boldsymbol{x}}(\boldsymbol{x}(t), \boldsymbol{u}(t), \boldsymbol{p}) \Delta \boldsymbol{x}(t)+\frac{\partial \boldsymbol{f}}{\partial \boldsymbol{p}}(\boldsymbol{x}(t), \boldsymbol{u}(t), \boldsymbol{p}) \Delta \boldsymbol{p} \\
& \Delta \boldsymbol{y}(t)=\frac{\partial \boldsymbol{g}}{\partial \boldsymbol{x}}(\boldsymbol{x}(t), \boldsymbol{u}(t), \boldsymbol{p}) \Delta \boldsymbol{x}(t)+\frac{\partial \boldsymbol{g}}{\partial \boldsymbol{p}}(\boldsymbol{x}(t), \boldsymbol{u}(t), \boldsymbol{p}) \Delta \boldsymbol{p}
\end{aligned}
$$

for the deviations of the state vector and the output vector from nominal values due to parameter changes $\Delta \boldsymbol{p}$. The state sensitivity function $(\partial \boldsymbol{x} / \partial \boldsymbol{p})(t)$ and the output sensitivity function $(\partial \boldsymbol{y} / \partial \boldsymbol{p})(t)$ are given by the following equations:

$$
\begin{aligned}
& \frac{\partial \dot{\boldsymbol{x}}}{\partial \boldsymbol{p}}(t)=\frac{\partial \boldsymbol{f}}{\partial \boldsymbol{x}}(\boldsymbol{x}(t), \boldsymbol{u}(t), \boldsymbol{p}) \frac{\partial \boldsymbol{x}}{\partial \boldsymbol{p}}+\frac{\partial \boldsymbol{f}}{\partial \boldsymbol{p}}(\boldsymbol{x}(t), \boldsymbol{u}(t), \boldsymbol{p}) \\
& \frac{\partial \boldsymbol{y}}{\partial \boldsymbol{p}}(t)=\frac{\partial \boldsymbol{g}}{\partial \boldsymbol{x}}(\boldsymbol{x}(t), \boldsymbol{u}(t), \boldsymbol{p}) \frac{\partial \boldsymbol{x}}{\partial \boldsymbol{p}}+\frac{\partial \boldsymbol{g}}{\partial \boldsymbol{p}}(\boldsymbol{x}(t), \boldsymbol{u}(t), \boldsymbol{p})
\end{aligned}
$$

These equations can be solved if the $\boldsymbol{x}(t)$ and $\boldsymbol{u}(t)$ in the initial model are known. Consequently, equations (30a), (30b), (31a) and (31b) must be solved simultaneously.

Alternatively, a pseudo bond graph can be constructed in which each bond instead of the incremental power variables carries the sensitivities of the power variables. For instance, differentiation of the constitutive relation

$$
e_{R}(t)=\Phi_{R}\left(f_{R}(t), p\right)
$$

of a non-linear one-port resistor with a single parameter $p$ yields

$$
\frac{\partial e_{R}(t)}{\partial p}=\frac{\partial \Phi_{R}}{\partial f_{R}}\left(f_{R}(t), p\right) \frac{\partial f_{R}(t)}{\partial p}+\frac{\partial \Phi_{R}}{\partial p}\left(f_{R}(t), p\right)
$$

If the sensitivities $\partial e_{R} / \partial p$ and $\partial f_{R} / \partial p$ are used as bond variables the pseudo bond graph corresponding to equation (33) has the same structure as that in Fig. 13.

A pseudo bond graph approach has been proposed by Cabanellas et al. [2] and has been extended by
Gawthrop [11]. The relation between the initial bond graph and the incremental bond graph is depicted in Fig. 15. The (time domain) solution of the equations of the initial model provides values for the vector of system output variables, $\boldsymbol{y}$, and the vector of state variables, $\boldsymbol{x}$. In addition, generalized displacements may or must be computed (vector $\boldsymbol{q}$ ). Some power variables in the initial bond graph depending on state and input variables are input to modulated sinks that link the initial bond graph in a unilateral direction to the incremental bond graph. The equations derived from the incremental bond graph determine finite deviations of the state and the output vector, if finite parameter changes are known; i.e. perturbed power variables can be computed. In the same way that generalized displacements may be computed from the initial bond graph model, sensitivities can be computed, if in the equations of the incremental bond graph deviations of the power variables from nominal values are replaced by sensitivities [cf. equations (30a) and (30b)].

\section{Example: a simple model containing a linear multiport}

Consider a simple circuit containing a transformer with mutually interacting coils (Fig. 16). The full circles

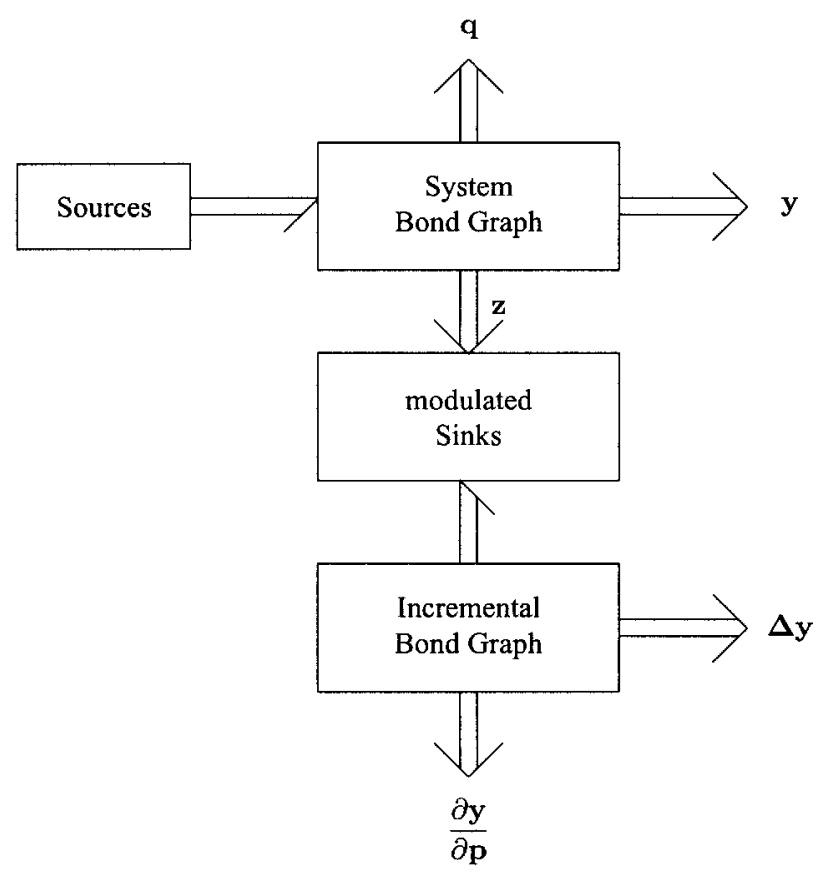

Fig. 15 Relation between initial and incremental bond graph

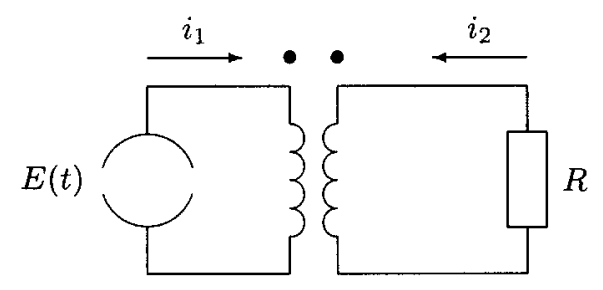

Fig. 16 Transformer with mutual interacting coils 
above the coils denote their relative orientation. In this case, both currents $i_{1}$ and, $i_{2}$ enter the ends with full circles and their mutual inductance coefficient $M_{12}$ is positive. Such a transformer is appropriately described by means of a two-port I field as depicted in Fig. 17. Its constitutive equation is

$$
\left[\begin{array}{l}
i_{1} \\
i_{2}
\end{array}\right]=\left(\begin{array}{cc}
\frac{1}{L_{1}} & \frac{1}{M_{12}} \\
\frac{1}{M_{12}} & \frac{1}{L_{2}}
\end{array}\right)\left[\begin{array}{l}
\lambda_{1} \\
\lambda_{2}
\end{array}\right]
$$

In equation (34), $L_{1}$ and $L_{2}$ denote the self-inductance coefficients of the two coils, while $M_{12}$ is the mutualinductance coefficient.

For the two flux linkages $\lambda_{1}$ and $\lambda_{2}$ the following state space equation is easily obtained:

$$
\left[\begin{array}{l}
\dot{\lambda}_{1} \\
\dot{\lambda}_{2}
\end{array}\right]=\underbrace{\left(\begin{array}{cc}
0 & 0 \\
-\frac{R}{M_{12}} & -\frac{R}{L_{2}}
\end{array}\right)}_{\mathbf{A}} \underbrace{\left[\begin{array}{l}
\lambda_{1} \\
\lambda_{2}
\end{array}\right]}_{\boldsymbol{x}}+\underbrace{\left(\begin{array}{l}
1 \\
0
\end{array}\right)}_{\mathbf{B}} \underbrace{[E]}_{\boldsymbol{u}}
$$

If the load current $i_{1}$ on the voltage source is chosen as an output variable $y$, the matrices $\mathbf{C}$ and $\mathbf{D}$ become

$$
\left[i_{1}\right]=\underbrace{\left(\frac{1}{L_{1}} \frac{1}{M_{12}}\right.}_{\mathbf{C}})\left[\begin{array}{l}
\lambda_{1} \\
\lambda_{2}
\end{array}\right]
$$

and $\mathbf{D}=\mathbf{0}$. Hence,

$$
s \mathbf{I}-\mathbf{A}=\left(\begin{array}{cc}
s & 0 \\
\frac{R}{M_{12}} & s+\frac{R}{L_{2}}
\end{array}\right)
$$

Thus the characteristic polynomial is

$$
\Delta:=s^{2}+\frac{R}{L_{2}} s
$$

From equation (35) the matrix $\tilde{\mathbf{B}}$ is obtained according to equation (13a):

$$
\begin{aligned}
& \tilde{\mathbf{B}}=\frac{\partial}{\partial\left(L_{1}, L_{2}, M_{12}, R\right)}\left[\begin{array}{c}
E \\
-\frac{R}{M_{12}} \lambda_{1}-\frac{R}{L_{2}} \lambda_{2}
\end{array}\right] \\
& =\left(\begin{array}{cccc}
0 & 0 & 0 & 0 \\
0 & R \frac{\lambda_{2}}{L_{2}^{2}} & R \frac{\lambda_{1}}{M_{12}^{2}} & -i_{2}
\end{array}\right) \\
& S_{e} \frac{E=\dot{\lambda}_{1}}{i_{1}} \gamma_{1} \frac{\dot{\lambda}_{2}}{i_{2}} 1 \longmapsto \mathrm{R}: \mathrm{R}
\end{aligned}
$$

Fig. 17 Bond graph of the circuit in Fig. 16
Accordingly, the matrix $\tilde{\mathbf{D}}$ is

$$
\begin{aligned}
\tilde{\mathbf{D}} & =\frac{\partial}{\partial\left(L_{1}, L_{2}, M_{12}, R\right)}\left[\frac{\lambda_{1}}{L_{1}}+\frac{\lambda_{2}}{M_{12}}\right] \\
& =\left(\begin{array}{llll}
-\frac{\lambda_{1}}{L_{1}^{2}} & 0 & -\frac{\lambda_{2}}{M_{12}^{2}} & 0
\end{array}\right)
\end{aligned}
$$

With the above matrices, manual calculation of the sensitivity matrix $\mathbf{S}$ according to equation (12) is a lengthy procedure and is therefore not shown here. Alternatively, the incremental bond graph depicted in Fig. 18 is entered in CAMP-G, and MATLAB is employed to set up the sensitivity matrix from the data generated by CAMP-G. As can be seen from Fig. 18, the sinks in the incremental models of the I field and the resistor have been split up into a sink and a modulated transformer (see Fig. 3). The value of the sink is the change in the parameter, e.g. $\Delta L_{1}$, while the modulus of the transformer is the corresponding sensitivity, e.g. $\partial i_{1} / \partial L_{1}$. The incremental bond graph entered in CAMP-G can be seen partly from Fig. 19. (Other parts of the graph become visible by scrolling the bars on the left-hand side and on the bottom of the drawing window.) The bond graph preprocessor CAMP-G generates the MATLAB procedure campsym.m. In this file, comment signs are removed in appropriate places in order to identify $\Delta i_{1}$ as an output variable and to activate the calculation of the transfer functions matrix, i.e. the sensitivity matrix. Execution of the MATLAB procedure gives the results shown in Fig. 20 and Fig. 21. Taking into account that CAMP-G assigns numbers to the bonds of a bond graph instead of names for the power variables the system matrices generated by CAMP-G are the same as those that have been calculated manually [cf. equations (35) and (36)]. As can be seen from Fig. 21, the entries in the sensitivity matrix are lengthy expressions in which, for example, the transformer moduli $T_{i j}$ have to be replaced by the appropriate partial derivatives with respect to the parameters (see Fig. 18).

\section{CONCLUSION}

In this paper a bond graph based approach to the determination of first-order frequency domain sensitivities has been presented. Instead of representing constraints between sensitivities in a pseudo bond graph, it is proposed to set up a true power bond graph for the deviations $\Delta e$ and $\Delta f$ from the nominal power variables that are due to small parameter changes. The associated bond graph for the incremental power variables is obtained systematically by replacing each bond graph element by a submodel that consists of standard bond graph elements. If only a certain sensitivity is of interest, it is sufficient to replace the affected element by its associated incremental model. Although the product of the incremental power variables is indeed power, in fact it 


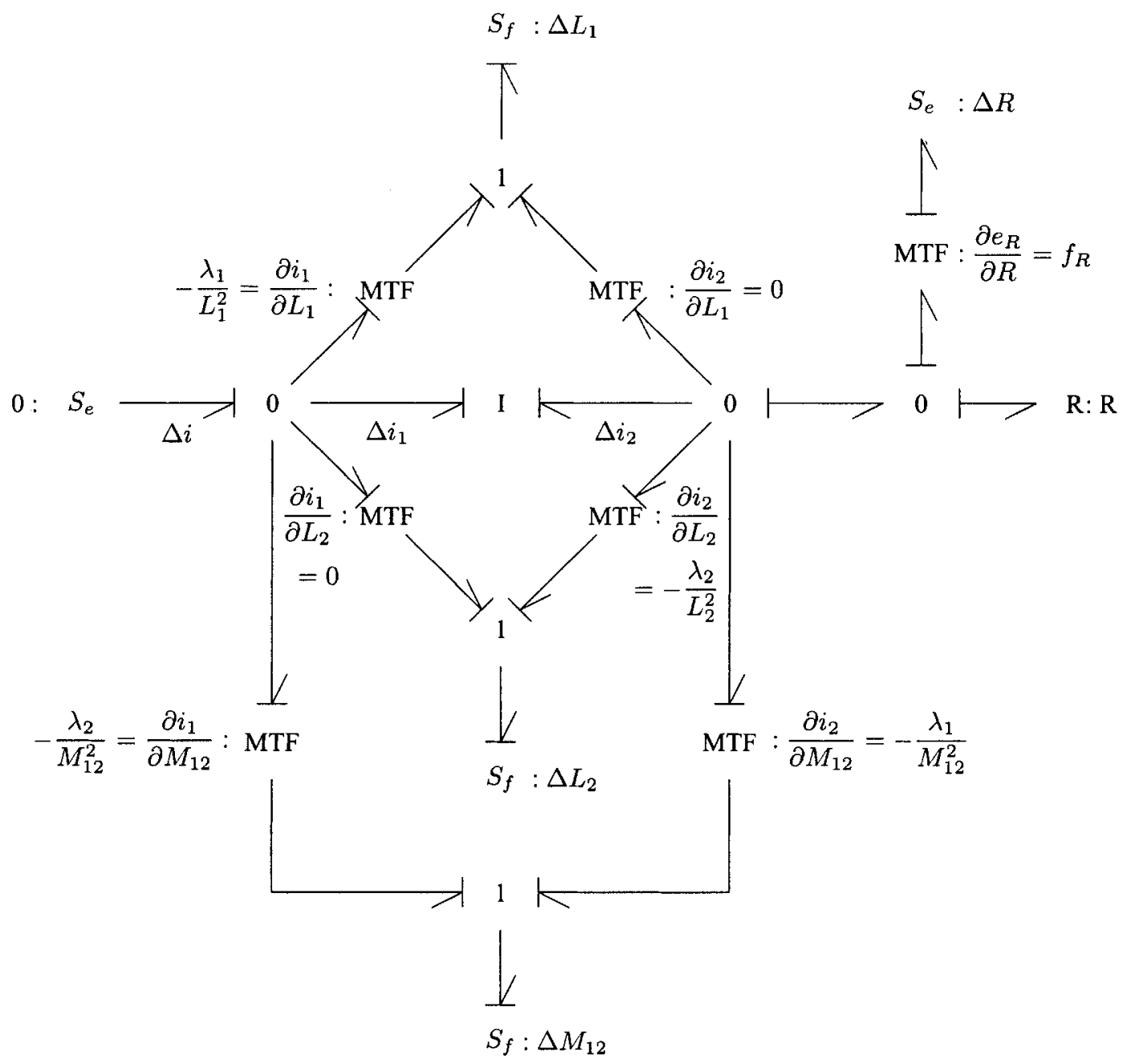

Fig. 18 Incremental bond graph of the transformer circuit in Fig. 16

is only a part of the change in power due to parameter changes. Apart from additional sinks the structure of the original bond graph is retained by the incremental bond graph. Owing to the additional sinks, a submodel that replaces a bond graph element does not have all properties of that element; i.e. a submodel replacing a resistor is not passive and a submodel replacing a transformer is not power conservative. However, the additional sinks in the incremental model of an element do not affect causalities. Moreover, equations can be derived from the incremental bond graph in the same way as they are derived from the initial bond graph. The sensitivities to be determined are just the coefficients in these linear equations, if the parameter changes are assumed to be infinitesimally small. The approach is applicable to bond graphs containing non-linear multiport elements. In the latter case the incremental bond graph model is linear time variant. Consequently, since the Laplace transform cannot be used any longer, the equations of the original model and those of the sensitivity model must be solved simultaneously in time domain. Finally, since the construction of the associated incremental bond graph is fully systematic, it could be performed automatically in a bond graph modelling package and could be completely hidden from those modellers who just ask for output of sensitivities of interest. This is only a matter of implementation. Incremental models of commonly used (non-linear) elements could be stored in a library. If the modeller requests sensitivities, the software could pick the incremental submodels needed in a way that is transparent for the user. In the case of linear time-invariant systems a tool for symbolic mathematical calculation, e.g. MATLAB, could be exploited to provide sensitivities in symbolic form. In the non-linear case the simulation part of the software must solve simultaneously the equations of the initial bond graph model and the equations that determine the sensitivities.

In the linear case it might be argued that there is no need for an associated sensitivity bond graph because sensitivities may be obtained by symbolic differentiation of transfer functions. In fact, if the Laplace transforms of a system input $u$ and an output variable $y$ are related by a transfer function $F(s, \boldsymbol{p})$ and if $\boldsymbol{p}$ denotes a 


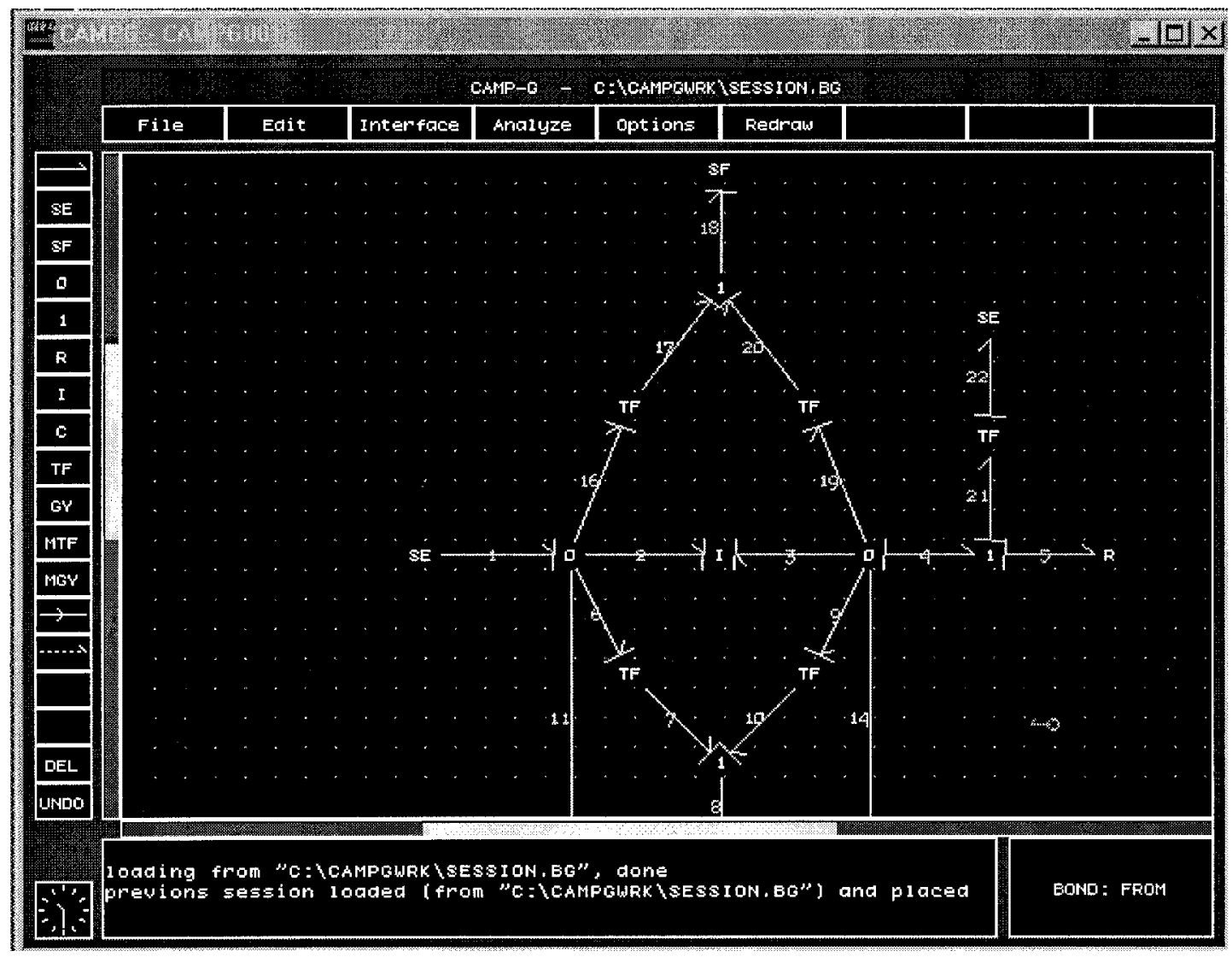

Fig. 19 Incremental bond graph of Fig. 18 entered in CAMP-G

\section{A MATRIX}

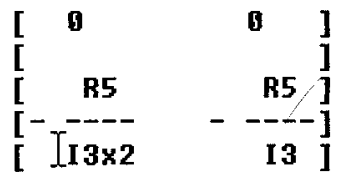

B MATRIX

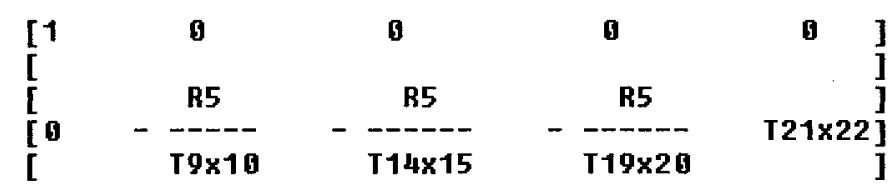

C MATRIX

$$
\left[\begin{array}{cc}
1 & 1
\end{array}\right]
$$

D MaTRIX

$$
\left[\begin{array}{ccccr}
{[} & 1 & 1 & 1 & ] \\
{[} & -16 \times 7 & T 11 \times 12 & T 16 \times 17 & ]
\end{array}\right]
$$

Fig. 20 System matrices set up by CAMP-G/MATLAB 


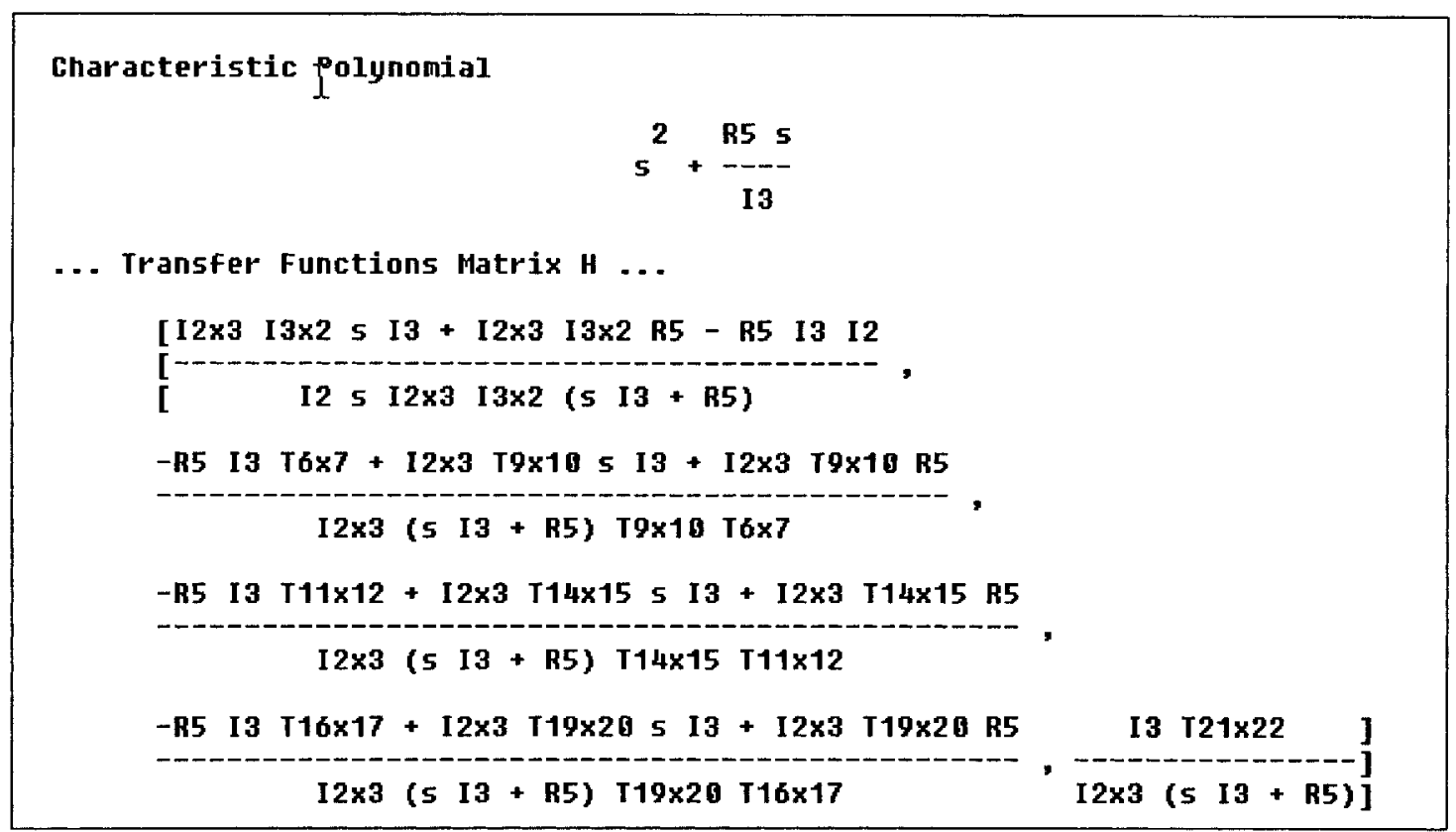

Fig. 21 Sensitivity matrix set up by CAMP-G/MATLAB

parameter vector, then the sensitivity of $\mathscr{L} y$ with respect to $p_{i}$ is given by

$$
\frac{\partial \mathscr{L} y}{\partial p_{i}}=\frac{\partial F(s, \boldsymbol{p})}{\partial p_{i}} \mathscr{L} u
$$

Although $F$ is in general a non-linear function of the parameters $p_{j}(j=1, \ldots, r)$, the differentiation may be carried out symbolically. In MATLAB, for example, jacobian could be used. However, for a user of the bond graph modelling approach the incremental bond graph is a valuable tool since it provides insight into the effects of parameter changes of the model. Ultimately, in the case of linear time-invariant models all unnormalized sensitivities may be obtained in symbolic form in one step as has been shown in this paper by using MATLAB for the derivation of the general transfer function matrix from the incremental bond graph.

\section{ACKNOWLEDGEMENTS}

This work was accomplished before the 2001 International Conference on Bond Graph Modeling and Simulation (ICBGM'01). The first author would like to thank Professor Peter Gawthrop from the University of Glasgow, for useful discussions and valuable hints during the conference. He would like to thank also Professor Geneviève Dauphin-Tanguy and Casimir S. Kam for stimulating discussions in a friendly atmosphere during his visit to the École Centrale de Lille, France. These discussions helped to obtain a clearer view of some details.

\section{REFERENCES}

1 Director, S. W. and Rohrer, R. A. The generalized adjoint network and network sensitivity. IEEE Trans. Circuit Theory, August 1969, CT-16, 318-323.

2 Cabanellas, J. M., Félez, J. and Vera, C. A formulation of the sensitivity analysis for dynamic systems optimization based on pseudo bond graphs. In Proceedings of the 1995 International Conference on Bond Graph Modeling and Simulation (ICBGM'95), Simulation Series, Vol. 27, No. 1 (Eds F. E. Cellier and J. J. Granda), Las Vegas, Nevada, January 1995, pp. 135-144 (Society for Computer Simulation, San Diego, California).

3 Roe, P. H. and Thoma, J. U. A new bond graph approach to sensitivity analysis. In Proceedings of the 3rd MATHMOD Vienna, IMACS Symposium on Mathematical Modelling (Eds I. Troch and F. Breitenecker), 2000, pp. 743-746 (ARGESIM, Vienna).

4 Borutzky, W. and Granda, J. Determining sensitivities from an incremental true bond graph. In Proceedings of the 2001 International Conference on Bond Graph Modeling and Simulation (ICBGM 2001), Simulation Series, Vol. 33, No. 1 (Eds J. J. Granda and G. Dauphin-Tanguy), 2001, pp. 3-8 (Society for Computer Simulation, San Diego, California).

5 Rosenberg, R. C. State-space formulation for bond graph models of multiport systems. J. Dynamic Systems, Measmt, Control, March 1971, pp. 35-40.

6 Granda, J. and Reus, J. New developments in bond graph modeling software tools: the computer aided modeling program CAMP-G and MATLAB. In Proceedings of the IEEE International Conference on Systems, Man, and Cybernetics, October 1997 (IEEE, New York).

7 Brown, F. T. Direct application of the loop rule to bond graphs. J. Dynamic Systems, Measmt, Control, September 1992, 253-261. 
8 Granda, J. Computer generated transfer functions CAMP-G: interface to MATLAB and SIMULINK. In Proceedings of the 1999 International Conference on Bond Graph Modeling and Simulation (ICBGM'99), Simulation Series, Vol. 31, No. 1, San Francisco, California, January 1999, pp. 129-135 (Society for Computer Simulation, San Diego, California).
9 CAMP-G-User's Manual, 1999 (Cadsim Engineering, Davis, California); see also http:/www.bondgraph.com.

10 Karnopp, D. Power and energy in linearized physical systems. J. Franklin Inst., 1977, 303, 85-98.

11 Gawthrop, P. Sensitivity bond graphs. J. Franklin Inst., 2000, 337, 907-922.

This publication is with permission of the rights owner freely accessible at https://nbn-resolving.org/urn:nbn:de:hbz:1044-opus-1422

due to an Alliance licence and a national licence (funded by the DFG, German Research Foundation) respectively. You are free to use this Item in any way that is permitted by the copyright and related rights legislation that applies to your use. For other uses you need to obtain permission from the rights-holder(s). 